\title{
Uncovering the high-scale Higgs singlet model
}

\author{
Sally Dawson $\odot,{ }^{1}$ Pier Paolo Giardino $\odot,{ }^{2}$ and Samuel Homiller ${ }^{3}$ \\ ${ }^{1}$ Department of Physics, Brookhaven National Laboratory, Upton, New York 11973, USA \\ ${ }^{2}$ Instituto Galego de Física de Altas Enerxías, Universidade de Santiago de Compostela, \\ 15782 Santiago de Compostela, Galicia, Spain \\ ${ }^{3}$ Department of Physics, Harvard University, Cambridge, Massachusetts 02138, USA
}

(Received 10 February 2021; accepted 15 March 2021; published 19 April 2021)

\begin{abstract}
The scalar singlet model extends the Standard Model with the addition of a new gauge singlet scalar. We reexamine the limits on the new scalar from oblique parameter fits and from a global fit to precision electroweak observables and present analytic expressions for our results. For the case when the new scalar is much heavier than the weak scale, we map the model onto the dimension-six Standard Model effective field theory and review the allowed parameter space from unitarity considerations and from the requirement that the electroweak minimum be stable. A global fit to precision electroweak data, along with LHC observables, is used to constrain the parameters of the high scale singlet model, and we determine the numerical effects of performing the matching at both tree level and one loop.
\end{abstract}

DOI: 10.1103/PhysRevD.103.075016

\section{INTRODUCTION}

The Higgs singlet model [1-7] has been extensively studied as a simple extension of the Standard Model (SM) containing only one new particle. Depending on the potential parameters, the model can lead to a first order electroweak phase transition [8-18], making it highly motivated in addressing the problem of baryogenesis. It can also arise as the limiting case of many interesting models addressing the hierarchy problem $[19,20]$ or even dark matter [21-27]. When the mass of the new scalar becomes much larger than the weak scale, the theory can be mapped onto an effective field theory. The utility and simplicity of the model thus makes it an ideal candidate for exploring the limits of an effective field theory framework in reproducing the features of the underlying UV models [28-35].

In the full UV complete singlet model, restrictions on the parameters can be found from fits to precision electroweak observables as well as LHC data. These limits can then be compared with limits found in the context of a low energy effective field theory. We consider an effective field theory in which the SM Higgs doublet is constrained to be an $S U$ (2) doublet, the Standard Model effective field theory (SMEFT). At tree level, the singlet model generates only

Published by the American Physical Society under the terms of the Creative Commons Attribution 4.0 International license. Further distribution of this work must maintain attribution to the author(s) and the published article's title, journal citation, and DOI. Funded by SCOAP ${ }^{3}$. two SMEFT coefficients when matched at the UV scale $[31,36]$. The aim of this work is to examine to what extent the extraction of SMEFT coefficients from global fits at the weak scale gives information on the parameters of the UV complete singlet model $[28,32-34,37-39]$. The focus is on understanding the numerical importance of various choices made when performing the low energy fits and to this end, we implement both tree and one-loop matching [40-42] at the UV scale. We find that the effects of the one-loop matching are typically rather small. Effects of $\mathcal{O}(10 \%)$ can be obtained only for rather large values of certain dimensionless parameters in the Lagrangian.

Section II contains a recap of the model and restrictions on the model parameters from unitarity and the minimization of the potential. Analytic results for electroweak precision observables in the singlet model are found in Sec. III along with a comparison between a global fit to electroweak precision observables (EWPOs) and a fit to the oblique parameters, and restrictions from unitarity and the minimization of the potential are in Sec. IV. The SMEFT matching with the singlet model at both tree and loop level is studied in Sec. V, and a global fit to electroweak precision observables, Higgs, and diboson data is presented. Section VI has some conclusions.

\section{BASICS}

The singlet model we consider contains the SM Higgs doublet, $\Phi$, and a scalar gauge singlet, $S$. The most general scalar potential is 


$$
\begin{aligned}
V(\Phi, S)= & -\mu_{H}^{2} \Phi^{\dagger} \Phi+\lambda_{H}\left(\Phi^{\dagger} \Phi\right)^{2}+\frac{m_{\xi}}{2} \Phi^{\dagger} \Phi S+\frac{\kappa}{2} \Phi^{\dagger} \Phi S^{2} \\
& +t_{S} S+\frac{M^{2}}{2} S^{2}+\frac{m_{\zeta}}{3} S^{3}+\frac{\lambda_{S}}{4} S^{4}
\end{aligned}
$$

The parameters can be redefined such that $\langle S\rangle \equiv x=0$. After spontaneous symmetry breaking, the two neutral scalars, $\Phi_{0}$ and $S$, mix to form the physical scalars, $h$ and $H$,

$$
\begin{gathered}
h=\cos \theta \Phi_{0}+\sin \theta S, \\
H=-\sin \theta \Phi_{0}+\cos \theta S,
\end{gathered}
$$

with the physical masses, $m_{h}=125.1 \mathrm{GeV}$ and $M_{H}$. The parameters of the model can be taken as

$m_{h}, M_{H}, v=246 \mathrm{GeV}, \sin \theta, x=0, \kappa, m_{\zeta}, \lambda_{S}$.

The other parameters of the Lagrangian are determined in the singlet model by ${ }^{1}$

$$
\begin{aligned}
m_{\xi} & =\frac{m_{h}^{2}-M_{H}^{2}}{v} \sin 2 \theta, \\
M^{2} & =m_{h}^{2} \sin ^{2} \theta+M_{H}^{2} \cos ^{2} \theta-\frac{\kappa}{2} v^{2}, \\
\lambda_{H} & =\frac{m_{h}^{2} \cos ^{2} \theta+M_{H}^{2} \sin ^{2} \theta}{2 v^{2}} .
\end{aligned}
$$

The $Z_{2}$ symmetric case has $m_{\zeta}=t_{S}=m_{\xi}=0$ and $x \neq 0$.

The couplings of $h$ to SM fermions and gauge bosons are suppressed relative to the SM Higgs couplings by a factor of $\cos \theta$, while the $H$ couplings are suppressed by $\sin \theta$. We can thus immediately find a trivial limit on $\cos \theta$ from Higgs production to SM particles $X$ (assuming no decays to invisible particles) ${ }^{2}$

$$
\cos ^{2} \theta=\mu \equiv \frac{\sigma \cdot \mathrm{BR}}{(\sigma \cdot \mathrm{BR})_{\mathrm{SM}}}
$$

Naively combining the combined ATLAS results with $80 \mathrm{fb}^{-1}$ [45] and the CMS combined limits with $139 \mathrm{fb}^{-1}[46]$,

$$
\mu[\mathrm{ATLAS}]=1.11_{-.08}^{+.09}, \quad \mu[\mathrm{CMS}]=1.02_{-.06}^{+.07},
$$

\footnotetext{
${ }^{1}$ We note that for $\kappa v^{2} \gg M^{2}$, the mass of the new scalar, $M_{H}$, comes from electroweak symmetry breaking and in this case the theory cannot be mapped onto the SMEFT $[42,43]$. Additionally, the kinematic distributions for $h h$ production in this limit are quite different from those where $M_{H}$ primarily depends on $M$ [44].

${ }^{2}$ If $2 M_{H}<m_{h}$ then the decay $h \rightarrow H H$ is allowed, altering the limit on $\cos \theta$.
}

we find at $95 \%$ C.L.

$$
|\sin \theta|<0.2 \text { for } m_{h}<2 M_{H} \text {. }
$$

For $m_{h}>2 M_{H}$, the naive limit of Eq. (7) does not apply because the $h$ decays to $H H$ must be included and this branching ratio is sensitive to the other parameters of the scalar potential. This simple limit can be complemented by direct search limits for a heavy Higgs boson [47] through the process $g g \rightarrow H \rightarrow W^{+} W^{-}$. In this case a comprehensive analysis including Higgs width effects and interference effects is needed [48-50] and for $M_{H} \sim$ $1 \mathrm{TeV}$ the limits depend on the width of the heavy Higgs boson which in turn is sensitive to all of the parameters of the potential. Limits on the singlet model from resonant double Higgs production are beginning to be competitive with those from single Higgs production for $M_{H} \lesssim$ $700 \mathrm{GeV}$ [51], although our primary focus here will be on $M_{H} \sim(1-2) \mathrm{TeV}$.

\section{RESTRICTIONS ON MODEL PARAMETERS}

The parameters of the singlet model can be limited by a fit to the $Z$ - and $W$-pole observables (we term this the EWPO fit):

$M_{W}, \quad \Gamma_{W}, \quad \Gamma_{Z}, \quad \sigma_{h}, \quad R_{b}, \quad R_{c}, \quad R_{l}, \quad A_{F B, b}$,

$A_{F B, c}, \quad A_{F B, l}, \quad A_{b}, \quad A_{c}, \quad A_{l}$.

The SM results for these observables are well known [52,53]. In a previous study, Ref. [54], we computed the limits on the coefficients of an effective field theory that result from a fit to the observables of Eq. (8) computed to next-to-leading order (NLO) in both QCD and electroweak interactions, and we apply an identical calculational framework here. The observables and SM theory numbers used in the current study can be found in Table III of Ref. [54]. We take as our input parameters $G_{\mu}=$ $1.1663787(6) \times 10^{-5} \mathrm{GeV}^{-2}, \quad M_{Z}=91.1876 \pm .0021 \mathrm{GeV}$, $1 / \alpha=137.035999139(31), \quad \Delta \alpha_{\mathrm{had}}^{(5)}=0.02764 \pm 0.00009$, $\alpha_{s}\left(M_{Z}\right)=0.1181 \pm 0.0011, m_{h}=125.10 \pm 0.14 \mathrm{GeV}$, $m_{b}=4.18 \mathrm{GeV}$, and $M_{t}=172.9 \pm 0.5 \mathrm{GeV}$.

The one-loop relation between the Fermi constant $G_{\mu}$ and the vacuum expectation value $v$ is, as usual,

$$
G_{\mu}=\frac{1}{\sqrt{2} v^{2}}(1+\Delta r)
$$

where

$$
\Delta r=\Delta r_{\mathrm{SM}}+\Delta r_{\text {singlet }}
$$


In computing $\Delta r$, we use $\hat{M}_{W}^{2} \equiv\left(M_{Z}^{2} / 2\right)\left(1+\sqrt{1-\frac{\sqrt{8} \pi \alpha}{G_{\mu} M_{Z}^{2}}}\right)$ calculated from our inputs. For simplicity, we define $h \equiv m_{h}^{2}$, $H \equiv M_{H}^{2}, z \equiv M_{Z}^{2}$, and $w \equiv \hat{M}_{W}^{2}$ and obtain the simple form ${ }^{3}$

$$
\begin{aligned}
\Delta r_{\text {singlet }} & =\Delta r_{\text {singlet }}(h, H)-\Delta r_{\text {singlet }}(H, h), \\
\Delta r_{\text {singlet }}(h, H) & =\frac{\sqrt{2} \sin ^{2} \theta G_{\mu}}{16 \pi^{2}}\left\{-\frac{h}{2}+\frac{3 w A_{0}(h)}{(h-w)}+\frac{3 w h A_{0}(w)}{(H-w)(h-w)}\right\} .
\end{aligned}
$$

We find the one-loop prediction for $M_{W}$ in the singlet model,

$$
\begin{aligned}
M_{W}= & M_{W}^{\mathrm{SM}}+F_{W}(h, H)-F_{W}(H, h), \\
F_{W}(h, H)= & \frac{\alpha \sin ^{2} \theta}{8 \pi M_{W}}\left\{\frac{h z}{24(2 w-z)}+\frac{A_{0}(h)}{12(h-w) w(2 w-z)}\left(h w(w-4 z)+12 w^{2} z+h^{2}(z-w)\right)\right. \\
& +\frac{h z A_{0}(w)}{12(h-w) w(H-w)(z-w)(2 w-z)}\left((2 w-z)[h H-w(h+H)]+w^{2}(8 z-7 w)\right) \\
& \left.+\frac{h w A_{0}(z)}{12(z-w)(z-2 w)}-\frac{z B_{0}(w, h, w)}{12 w(w-z)}\left(h^{2}-4 h w+12 w^{2}\right)+\frac{w B_{0}(z, h, z)}{12(z-2 w)(z-w)}\left(h^{2}-4 h z+12 z^{2}\right)\right\} .
\end{aligned}
$$

This is in agreement with Ref. [56]. For a massless $b$ quark, the total $W$ decay width is

$$
\begin{aligned}
\Gamma_{W}= & \Gamma_{W}^{\mathrm{SM}}+G_{W}(h, H)-G_{W}(H, h), \\
G_{W}(h, H)= & -\frac{3 \sqrt{w} G_{F}^{2} \sin ^{2} \theta}{32 \pi^{3}}\left\{\frac{w h\left(-h H-8 w^{2}+4 w h+4 w H\right)}{2(h-4 w)(H-4 w)}-\frac{A_{0}(h)}{h(h-4 w)(h-w)}\left(h^{4}-7 h^{3} w+21 h^{2} w^{2}-32 h w^{3}+8 w^{4}\right)\right. \\
& +\frac{h A_{0}(w)}{(h-4 w)(h-w)(H-w)(H-4 w)}\left(4 w^{2}\left(H^{2}+h^{2}\right)-5 w h H(H+h)\right. \\
& \left.\left.+h^{2} H^{2}-4 w^{3}(H+h)+18 w^{2} h H-36 w^{4}\right)+\frac{B_{0}(w, h, w)}{(h-4 w)}\left(h^{3}-7 h^{2} w+20 h w^{2}-28 w^{3}\right)\right\} .
\end{aligned}
$$

Analytic expressions for the remaining observables of Eq. (8) are given in [57].

The finite $b$ mass contribution to $Z$ decays to bottom pairs is sensitive to the Higgs- $b$ Yukawa coupling and generates nonoblique contributions. We compute $R_{l}, R_{b}$, $R_{c}$, and $\Gamma_{Z}$ for $m_{b} \neq 0$ and find that the numerical effect is less than $\sim 2 \%$ for $M_{H}>20 \mathrm{GeV}$, rising to $\sim 5 \%$ for

${ }^{3}$ The function $A_{0}$ is defined as

$$
A_{0}\left(m^{2}\right)=\int \frac{d^{d} k}{(2 \pi)^{2}} \frac{1}{k^{2}-m^{2}},
$$

where we calculate in $d=4-2 \epsilon$ dimensions. $B_{0}$ is the Passarino-Veltman 2-point function,

$$
B_{0}\left(m_{1}^{2}, m_{2}^{2}, p^{2}\right)=\int \frac{d^{d} k}{(2 \pi)^{n}} \frac{1}{\left[k^{2}-m_{1}^{2}\right]\left[(k+p)^{2}-m_{2}^{2}\right]}
$$

The Passarino-Veltman functions are evaluated using QCDLOOPS [55].
$M_{H} \sim 10 \mathrm{GeV}$, justifying the neglect of $b$ mass effects in our fits.

We perform a fit, including correlations, to the observables of Eq. (8) to determine the maximum allowed value of $\sin \theta$ for a given value of $M_{H}$ including all one-loop contributions. It is of interest to compare the complete EWPO fit with the results using the oblique parameters only. Using the results of [58,59], we find that the differences between the Peskin-Takeuchi [60] variables in the Higgs singlet model and the SM take the form

$$
\begin{gathered}
\Delta S=\frac{\sin ^{2} \theta}{12 \pi}(\mathcal{G}(H, z)-\mathcal{G}(h, z)), \\
\Delta T=\frac{3 \sin ^{2} \theta}{16 \pi s_{W}^{2} c_{W}^{2}}(\mathcal{K}(H)-\mathcal{K}(h)), \\
\Delta S+\Delta U=\frac{\sin ^{2} \theta}{12 \pi c_{W}^{2}}(\mathcal{G}(H, w)-\mathcal{G}(h, w)),
\end{gathered}
$$



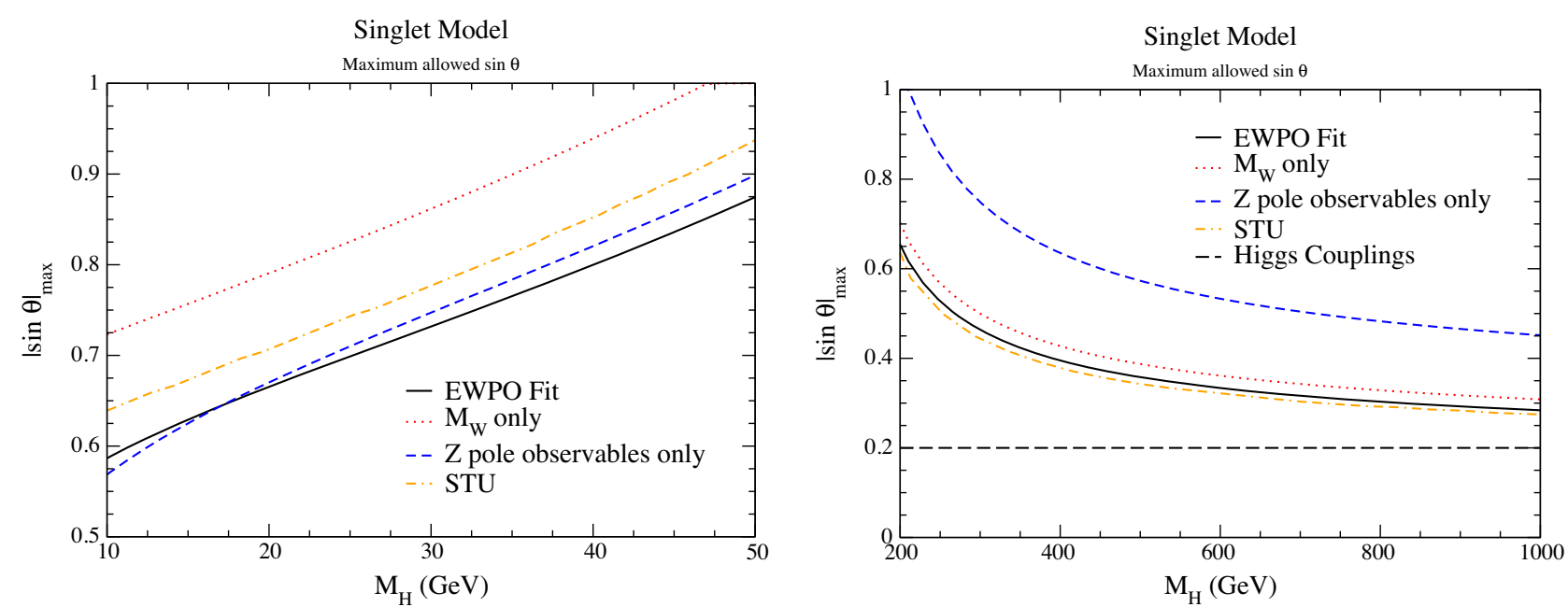

FIG. 1. Maximum allowed $\sin \theta$ in the Higgs singlet model at $95 \%$ confidence level based on fits to EWPO, the $W$ mass measurement only, the $Z$ pole observables only, and to the STU parameters as described in the text. A naive limit from the Higgs coupling measurements is shown on the right for comparison.

where $s_{W}^{2}=1-c_{W}^{2}=1-w / z$ is $\sin ^{2} \theta_{W}$, the electroweak mixing angle defined in the on-shell scheme, and we define

$\mathcal{K}(h)=h\left(\frac{(z-w)}{(h-w)(h-z)} A_{0}(h)+\frac{A_{0}(w)}{(h-w)}-\frac{A_{0}(z)}{(h-z)}\right)$,

$\mathcal{G}(h, z)=\frac{h}{2}+\mathcal{F}(h, z)\left(A_{0}(h)-A_{0}(z)-(h-z) B_{0}(z, h, z)\right)$,

$$
\mathcal{F}(h, z)=\frac{h^{2}-4 h z+12 z^{2}}{z(h-z)} .
$$

We fit to the values in [61]

$$
\begin{aligned}
& \Delta S=-0.01 \pm 0.10, \\
& \Delta T=0.02 \pm 0.12, \\
& \Delta U=0.02 \pm 0.11
\end{aligned}
$$

with the correlation matrix

$$
\rho=\left(\begin{array}{ccc}
1 . & 0.92 & -0.80 \\
0.92 & 1 . & -0.93 \\
-0.80 & -0.93 & 1 .
\end{array}\right)
$$

In Fig. 1 we report the results corresponding to different sets of observables:

(i) only $M_{W}$,

(ii) the $Z$ pole observables alone,

(iii) oblique parameters only, and

(iv) EWPOs given in Eq. (8).
The results for the fit to $M_{W}$ alone are in agreement with those of Ref. [47] and are a good approximation to the complete EWPO fit. The EWPO fit limits are in agreement with Ref. [39] after adjusting for the different input parameters. It is interesting that the current limits from Higgs couplings give better bounds for all $M_{H} \lesssim 1 \mathrm{TeV}$ as given in Eq. (7). The limits obtained from oblique parameters are in approximate agreement with those from the EWPO fit for a heavier second Higgs boson, although slightly different data sets and approximations were used in the numbers we fit to. ${ }^{4}$ For the case where the second Higgs is light, $M_{H}<m_{h}$, the limits obtained from the oblique parameters are not a good approximation of the complete EWPO fit. ${ }^{5}$

\section{THEORETICAL CONSTRAINTS}

In Sec. V, we will match the singlet model with a very heavy $H$ to the SMEFT. Before we do so, we consider the

\footnotetext{
${ }^{4}$ We find rough agreement with Refs. [12,39] (the differences can be explained by the different numerical values of the input parameters) and disagree with the oblique parameter limits of Fig. 1 of [62]. We note that the curve labeled "Exact Singlet" on the right-hand side of Fig. 1 of Ref. [28] is the $S T U$ result and has used a slightly different fit to the oblique parameters [63] from the PDG [61] results used here. The curve labeled Higgs in that plot is the prediction from fitting Higgs data within the context a SMEFT fit and thus differs from the SM Higgs coupling fit shown in Fig. 1.

${ }^{5}$ Of course, the new Higgs boson can also be searched for directly. There are numerous searches for $h \rightarrow H H$ with $M_{H}$ light which can be reinterpreted in terms of limits on the mass and mixing, e.g., $[64,65]$. In the non- $Z_{2}$ symmetric model that we consider, the branching ratio, $\mathrm{BR}(h \rightarrow H H)$, depends sensitively on all the parameters of the potential and so a simple limit in terms of $M_{H}$ and $\sin \theta$ is not possible.
} 


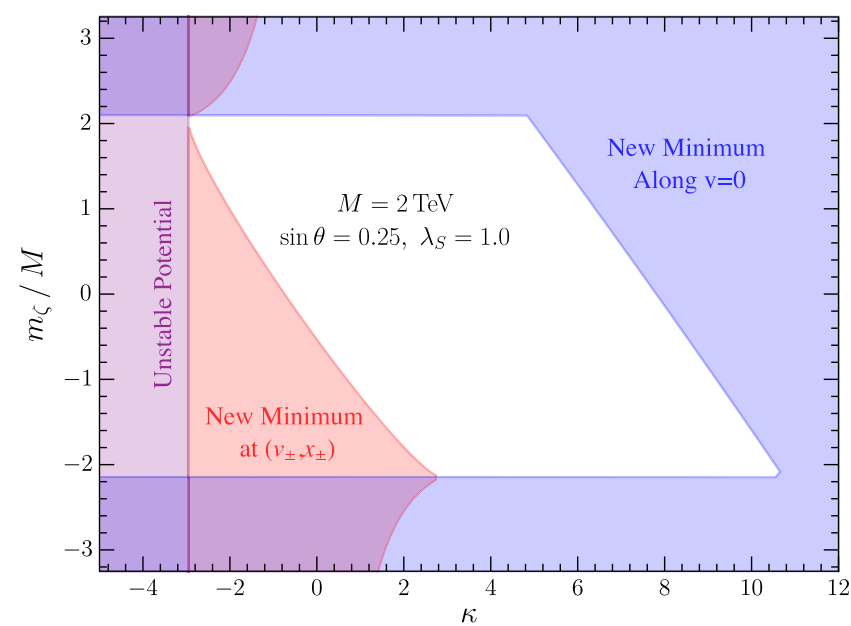

FIG. 2. Demonstration of the bounds from the appearance of other global minima in the $\kappa$ vs $m_{\zeta} / M$ plane for $M=2 \mathrm{TeV}$, $\sin \theta=0.25$, and $\lambda_{S}=1.0$ in the singlet model.

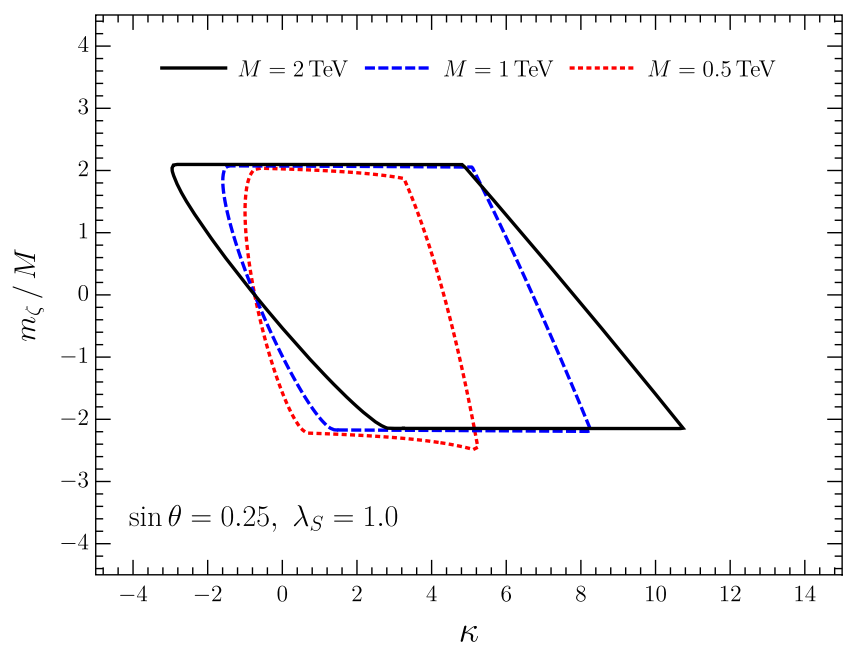

theoretical restrictions on the singlet model parameters that are relevant for the matching.

\section{A. Vacuum structure of the potential}

The first set of theoretical constraints on the singlet model come from requiring a suitable vacuum structure of the potential $[5,7,10,66]$. Demanding that the potential is stable at large field values leads to the requirement $\lambda_{H}, \lambda_{S}>0$, and $\kappa \geq-2 \sqrt{\lambda_{H} \lambda_{S}}$ [7], where $\lambda_{H}$ is determined by Eq. (4). Additional bounds result from requiring that the electroweak minimum be the global minimum of the potential. Following [7], we compute these bounds by finding all the extrema of the potential expanded around the electroweak vev as a function of $(v, x)$, and then checking whether or not the value of the potential at $(v=246 \mathrm{GeV}, x=0)$ is the global minimum.

The extrema of the potential can be divided into two classes: extrema where $v \neq 0$, and those where $v=0$. In

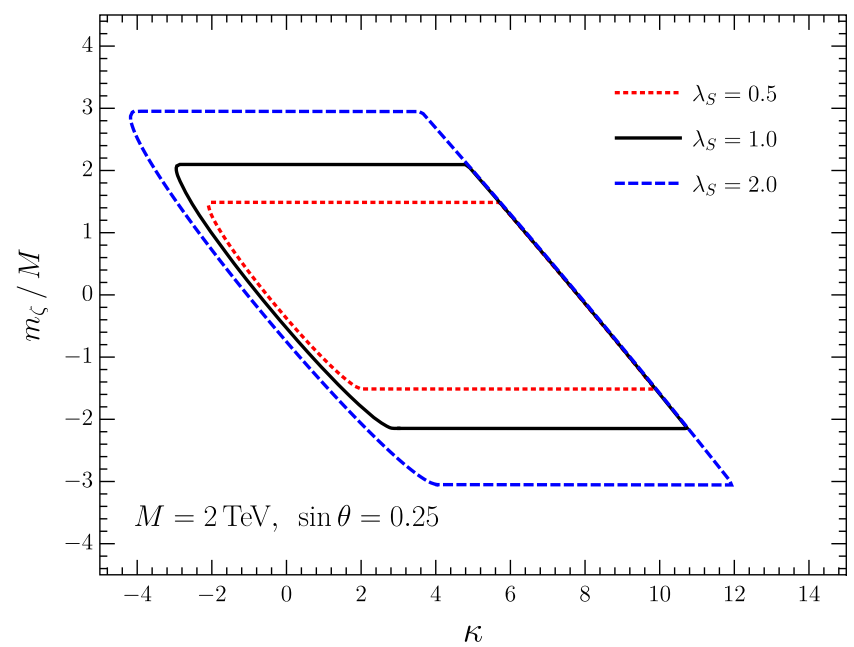

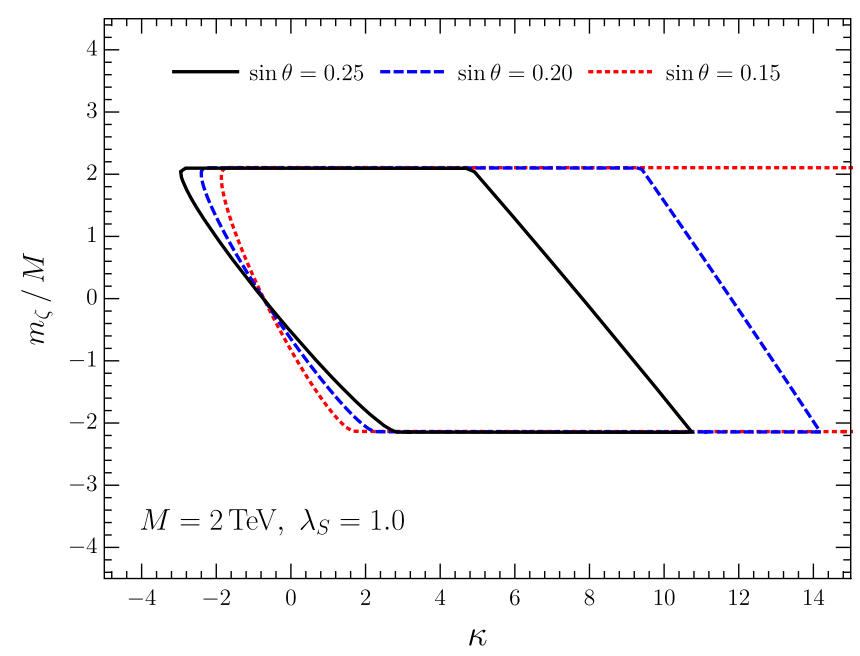

FIG. 3. Regions in the singlet model where the electroweak minimum is the global minimum of the potential as a function of $\kappa$ and $m_{\zeta} / M$, varying the other physical parameters. 
the former case, the new extrema are denoted $\left(v_{ \pm}, x_{ \pm}\right)$as in Ref. [7], and tend to bound lower values of $\kappa$. In the latter case, the extrema are denoted by $\left(0, x_{ \pm}^{0}\right)$, and these tend to limit both large values of $\kappa$ as well as large values of $m_{\zeta}$. An example of the vacuum structure is shown in Fig. 2, where we illustrate the regions excluded by the emergence of different global minima as well as the condition from vacuum stability. Figure 3 illustrates how these bounds change as a function of the physical parameters. In particular, we see that for larger masses, $M$, the bounds on $m_{\zeta} / M$ from the $v=0$ minima become constant as a function of $\kappa$, depending only on $\lambda_{S}$. It is interesting that quite large values of $\kappa$ are allowed in all scenarios. The upper bound on $m_{\zeta} / M$ never exceeds $\mathcal{O}(2-3)$.

\section{B. Unitarity}

The next set of theoretical constraints come from the requirements of tree-level perturbative unitarity [5,67-69]. The simplest constraints come from $h h \rightarrow h h$ and $H H \rightarrow$ $H H$ scattering, where the spin-0 partial waves in the high energy limit are

$$
\begin{aligned}
a_{0}( & h h \rightarrow h h)\left.\right|_{s \gg m_{h}^{2}} \\
& =-\frac{3}{16 \pi}\left(\lambda_{H} \cos ^{4} \theta+\kappa \sin ^{2} \theta \cos ^{2} \theta+\lambda_{S} \sin ^{4} \theta\right),
\end{aligned}
$$

$$
\begin{aligned}
&\left.a_{0}(H H \rightarrow H H)\right|_{s \gg m_{H}^{2}} \\
& \quad=-\frac{3}{16 \pi}\left(\lambda_{S} \cos ^{4} \theta+\kappa \sin ^{2} \theta \cos ^{2} \theta+\lambda_{H} \sin ^{4} \theta\right) .
\end{aligned}
$$

For $\sin \theta \ll 1$, requiring $\left|a_{0}\right|<1 / 2$ sets the bounds $\lambda_{S}, \lambda_{H} \lesssim 8 \pi / 3$. This bound on $\lambda_{H}$ indirectly bounds $M_{H}$ as a function of $\sin \theta$ :

$$
\begin{aligned}
M_{H}^{2} \sin ^{2} \theta & \lesssim \frac{16 \pi}{3} v^{2}-m_{h}^{2} \cos ^{2} \theta, \\
M_{H} & \lesssim 7 \mathrm{TeV} \text { for } \cos \theta=0.99
\end{aligned}
$$

The similar bound from $h H \rightarrow h H$ scattering only restricts $|\kappa| \lesssim 8 \pi$.

\section{ONE-LOOP MATCHING OF THE SINGLET MODEL TO SMEFT}

\section{A. One-loop matching}

When the mass of the heavy scalar is much larger than the weak scale and any relevant energy scales, the singlet model can be modeled by an effective field theory,

$$
\mathcal{L}_{\text {singlet }} \underset{M_{H} \rightarrow \infty}{\longrightarrow} \mathcal{L}_{\mathrm{SM}}+\sum_{i} \frac{C_{i}(M)}{M^{2}} \mathcal{O}_{i}^{(6)}+\ldots
$$

with coefficients matched to the singlet model at the high scale, $M$. We retain only the dimension- 6 operators, $\mathcal{O}_{i}^{(6)}$, and use the Warsaw basis [70] with the notation of Ref. [71].

The global fits of Ref. [28] were performed using tree-level matching at the scale $M{ }^{6}$ It is of interest to implement the one-loop matching for the case of the singlet model and examine the numerical impacts. The coefficients at the matching scale, $M$, generically take the form

$$
C_{i}(M)=c_{i}(M)+\frac{d_{i}(M)}{16 \pi^{2}}
$$

where $c_{i}(M)$ is the tree-level result and $d_{i}(M) /\left(16 \pi^{2}\right)$ is the one-loop contribution at the matching scale. When the renormalization group evolution to the low scale $\mu_{R}$ is included,

$C_{i}\left(\mu_{R}\right)=c_{i}(M)+\frac{d_{i}(M)}{16 \pi^{2}}+\frac{\gamma_{i j}}{32 \pi^{2}} c_{j}(M) \log \left(\frac{\mu_{R}^{2}}{M^{2}}\right)$.

In the case of the singlet model only two coefficients are generated at tree level $[31,36,69,72]$,

$$
\begin{gathered}
c_{H \square}=-\frac{m_{\xi}^{2}}{8 M^{2}}, \\
c_{H}=\frac{m_{\xi}^{2}}{8 M^{2}}\left(\frac{m_{\xi} m_{\zeta}}{3 M^{2}}-\kappa\right),
\end{gathered}
$$

with all other $c_{i}(M)=0$. However, there are many coefficients generated at one-loop at the matching scale, $M$ [40-42]. The majority of these coefficients are proportional to the tree-level coefficient, $c_{H \square}$. We use the shorthand $C_{H u} \rightarrow C_{H u}, C_{H c}, C_{H t}$, etc., and take $y_{u}=y_{c}=0, y_{t}=$ $M_{t} \sqrt{2} / v$ (similarly we set all other $y_{i}=0$ ) and we further assume that $C_{H q}^{(1)}, C_{H q}^{(3)}, C_{H l}^{(1)}$, and $C_{H l}^{(3)}$ are flavor diagonal and use an analogous shorthand. For convenience, we list the results of Ref. [40] in our notation ${ }^{7}$ :

\footnotetext{
${ }^{6}$ Ref. [29] noted that better agreement between the SMEFT and singlet model predictions for $h h$ production are obtained when the matching is performed at the physical mass, $M_{H}$. The one-loop matching would then contain terms proportional to $\log \left(M_{H} / M\right)$ that we have omitted.

${ }^{7}$ Since $y_{t}$ is the only nonzero Yukawa that we include, $O_{2 y}=y_{t}^{2} \bar{t} \bar{t} t$.
} 


$$
\begin{aligned}
d_{H D} & =\frac{31 g^{\prime 2}}{9} c_{H \square}, \\
d_{H W} & =-\frac{g^{2}}{6} c_{H \square}, \\
d_{H B} & =-\frac{g^{\prime 2}}{6} c_{H \square}, \\
d_{H W B} & =-\frac{g g^{\prime}}{3} c_{H \square}, \\
d_{H u} & =\frac{1}{108}\left(34 g^{\prime 2}-135 y_{u}^{2}\right) c_{H \square}, \\
d_{H d} & =\frac{1}{3} d_{H e}=\frac{2}{3} d_{H l}^{(1)}=-\frac{17 g^{\prime 2}}{108} c_{H \square}, \\
d_{H q}^{(1)} & =\frac{1}{216}\left(17 g^{\prime 2}+135 y_{u}^{2}\right) c_{H \square}, \\
d_{H q}^{(3)} & =\frac{1}{72}\left[17 g^{2}-45 y_{u}^{2}\right] c_{H \square}, \\
d_{H l}^{(3)} & =\frac{17 g^{2}}{72} c_{H \square}, \\
d_{2 y} & =-\frac{1}{3} c_{H \square} .
\end{aligned}
$$

The one-loop contribution $d_{t H}$ can be written in terms of $c_{H \square}$ and $C_{H}$ and is $d_{t H}=y_{t}\left[-\frac{1}{18}\left(45 y_{t}^{2}-31 g^{2}\right) c_{H \square}+\frac{3}{2} c_{H}-\frac{29}{3} \lambda c_{H \square}\right]$,

where in the SMEFT the physical Higgs mass is determined in terms of the potential parameters to $\mathcal{O}\left(v^{2} / M^{2}\right)$ by [71]

$$
\frac{m_{h}^{2}}{2 v^{2}}=\lambda_{H}\left(1+\frac{2 v^{2}}{M^{2}} c_{H \square}\right)-\frac{3}{2} \frac{v^{2}}{M^{2}} c_{H}
$$

and we define

$$
\begin{aligned}
\lambda & \equiv 2 \lambda_{H}\left(1+\frac{2 v^{2}}{M^{2}} c_{H \square}\right), \\
\lambda & =\frac{m_{h}^{2}}{v^{2}}+3 \frac{v^{2}}{M^{2}} c_{H}+\mathcal{O}\left(\frac{v^{4}}{M^{4}}\right),
\end{aligned}
$$

where we note that Ref. [40] absorbs the factor of $c_{H \square}$ into the definition of $\lambda$ used in the matching conditions, along with a relative factor of 2 in the definition of the quartic terms in the potential. ${ }^{8}$ Equation (31) represents the dimension-6 SMEFT limit of Eq. (4) for the relationship between the parameters of the potential and $m_{h}$.

Finally, the coefficients generated at tree level also receive one-loop corrections,

$$
\begin{aligned}
d_{H \square} & =-\frac{9}{2} \lambda c_{H \square}+\frac{31}{36}\left(3 g^{2}+g^{\prime 2}\right) c_{H \square}+\frac{3}{2} c_{H}+\delta d_{H \square}+\delta d_{H \square}^{\text {shift }}, \\
d_{H} & =\lambda\left[\left(\frac{62 g^{2}-336 \lambda}{9}\right) c_{H \square}+6 c_{H}\right]+\delta d_{H}+\delta d_{H}^{\text {shift }},
\end{aligned}
$$

where

$$
\begin{aligned}
\delta d_{H}= & -\frac{\kappa^{3}}{12}+\frac{m_{\xi}}{4 M^{2}}\left(m_{\xi}\left(9 \lambda^{2}-12 \kappa \lambda+\frac{11}{2} \kappa^{2}-3 \kappa \lambda_{S}\right)-\kappa^{2} m_{\zeta}\right) \\
& +\frac{m_{\xi}^{2}}{6 M^{4}}\left(\frac{m_{\xi}^{2}}{16}\left(39 \kappa-18 \lambda_{S}-36 \lambda\right)+m_{\xi} m_{\zeta}\left(9 \lambda-\frac{15}{2} \kappa+3 \lambda_{S}\right)+3 \kappa m_{\zeta}^{2}\right) \\
& +\frac{m_{\xi}^{3}}{12 M^{6}}\left(-\frac{1}{8} m_{\xi}^{3}-\frac{9}{8} m_{\xi}^{2} m_{\zeta}+3 m_{\xi} m_{\zeta}^{2}-2 m_{\zeta}^{3}\right), \\
\delta d_{H \square}=-\frac{\kappa^{2}}{24}+ & \frac{m_{\xi}}{12 M^{2}}\left(\frac{m_{\xi}}{2}\left(17 \kappa-\frac{27}{2} \lambda-18 \lambda_{S}\right)-5 \kappa m_{\zeta}\right)+\frac{m_{\xi}^{2}}{24 M^{4}}\left(\frac{13}{8} m_{\xi}^{2}-8 m_{\xi} m_{\zeta}+11 m_{\zeta}^{2}\right) .
\end{aligned}
$$

The terms of Eqs. (34) and (35) can be written in terms of $c_{H}, c_{H \square}$ along with $m_{\zeta}, \lambda_{S}$ and $\kappa,\left(m_{\xi}\right.$ can be written in terms of these parameters). The one-loop shift terms from canonically normalizing the Higgs kinetic energy are

$$
\begin{aligned}
& \delta d_{H}^{\text {shift }}=3 c_{H \square} c_{H}, \\
& \delta d_{H \square}^{\text {shift }}=2\left(c_{H \square}\right)^{2} .
\end{aligned}
$$

\footnotetext{
${ }^{8}$ We drop the $c_{H D}$ term in Eq. (31) since it does not occur in the singlet model.
} 


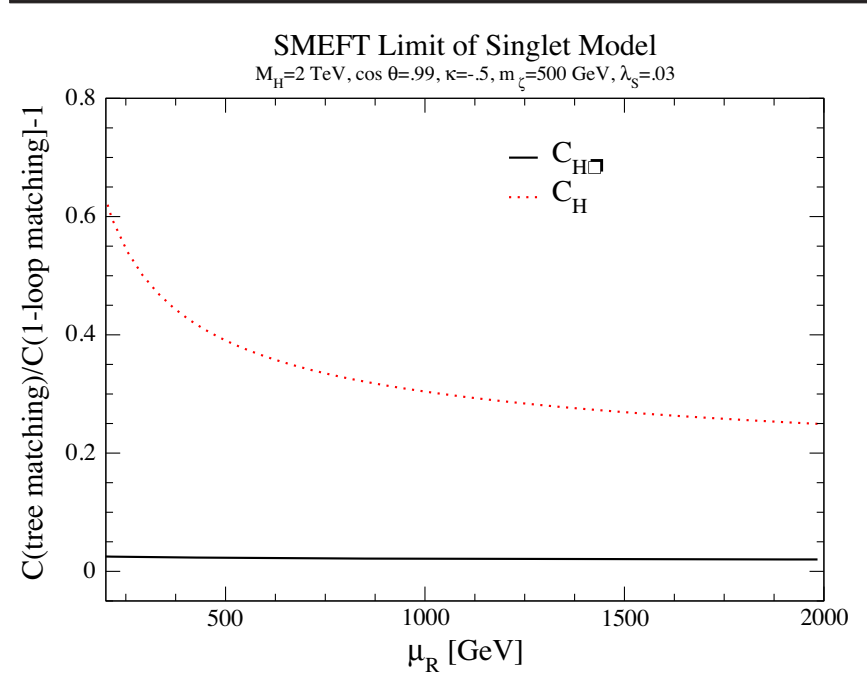

FIG. 4. Renormalization group evolution of coefficient functions from the matching scale, $M$, to $\mu_{R}$ when the matching is done at tree level and at one loop for coefficients that are generated at tree level. The coefficients are evaluated as a function of the running scale, $\mu_{R}$.

The one-loop shift terms are $\mathcal{O}\left(v^{4} / M^{4}\right)$ and can be neglected, since we consistently work to linear order in the coefficient functions.

After performing the one-loop matching at $M$, the renormalization group is used to evolve the coefficients to $M_{Z}$, where the resulting coefficients can be compared with data. ${ }^{9}$ The complete set of one-loop anomalous dimension matrices can be found in Refs. [73-75]. The inclusion of the one-loop matching makes a relatively minor difference in the evolution of $C_{H}$ and $C_{H \square}$, as seen in Fig. 4 where we evolve from $2 \mathrm{TeV}$ [note that $M_{H}$ is related to $M$ by Eq. (4)]. In Fig. 5, we show the effect of the oneloop matching on the evolution of $C_{H D}$. In this case, since $C_{H D}$ is zero at tree level, the contributions from the oneloop matching and the renormalization group running are of the same order of magnitude and the effects are more significant. In Fig. 6 we show the relative size of the oneloop matching compared to the tree-level matching as the matching scale $M$ is increased and the overall effects are between $10 \%$ and $30 \%$. The size of the effects for $C_{H \square}$ and $C_{H}$ increases dramatically as the matching scale rises over a few $\mathrm{TeV}$. This is due to the logarithmic running becoming large and in the case of $C_{H}$, the one-loop matching terms become of the same order as the tree-level terms, implying that the perturbative expansion is no longer valid.

\section{B. Global fit}

Following Ref. [28], we perform a global fit to the parameters of the non- $Z_{2}$ symmetric singlet model. At the

\footnotetext{
${ }^{9} \mathrm{~A}$ more consistent approach would employ the two-loop anomalous dimensions; however, these are not available for the SMEFT.
}

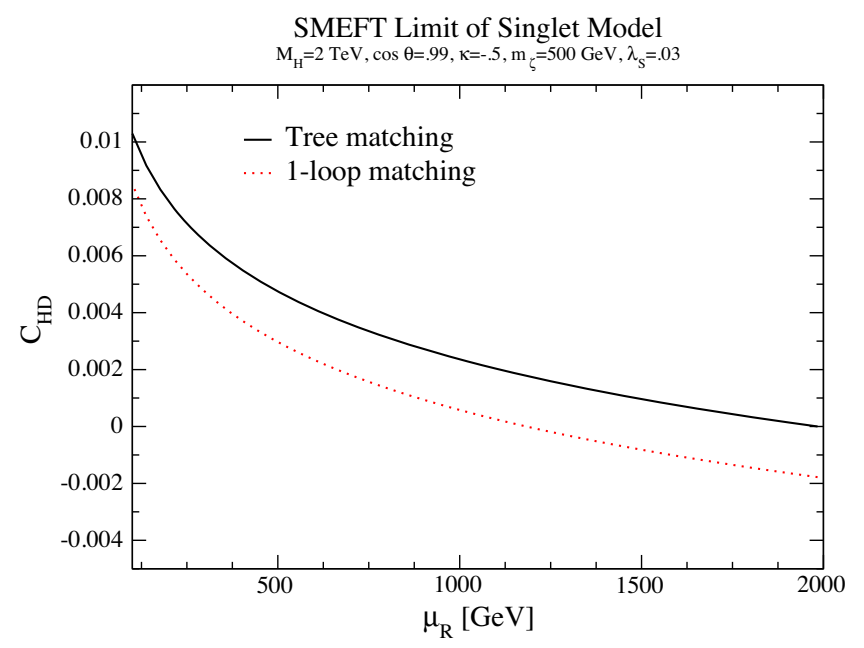

FIG. 5. Renormalization group evolution of the coefficient function from the matching scale, $M$, to $\mu_{R}$ for $C_{H D}$, which is generated only by the renormalization group running in the singlet model. $C_{H D}$ is evaluated as a function of the running scale, $\mu_{R}$.

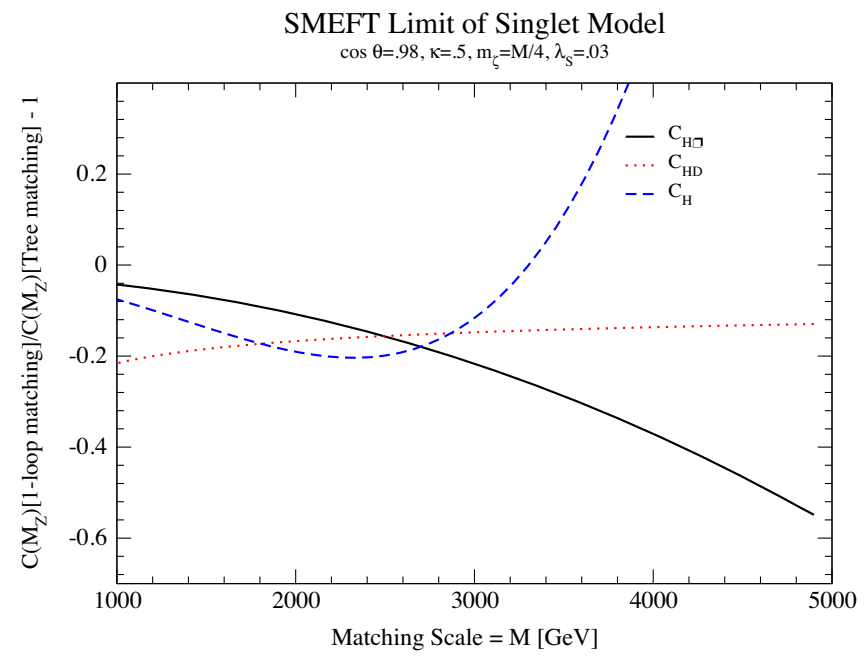

FIG. 6. Shift in the coefficient functions at $M_{Z}$ as a function of the matching scale, $M$, when the matching is done at tree level and at one loop.

matching scale, $M$, only the tree-level coefficients $c_{H}$ and $c_{H \square}$ are nonzero and other coefficients are generated at $M_{Z}$ from the renormalization group running. With tree-level matching, the results can be expressed in terms of $c_{H}(M)$ and $c_{H \square}(M)$. Using the one-loop matching at $M$ described in the previous section, additional coefficients are generated with a distinctive pattern. The one-loop matching introduces a dependence on three additional parameter combinations beyond those generated by the tree-level matching and we take as our five unknown input parameters $M_{H}$, $\sin \theta, m_{\zeta}, \lambda_{S}$, and $\kappa .{ }^{10}$ The matching scale, $M$, is then

\footnotetext{
${ }^{10}$ The results used to include the effects of $C_{H}$ require $\left|C_{H}\right| \lesssim$ $(5-6)(M / \mathrm{TeV})^{2}[76,77]$.
} 

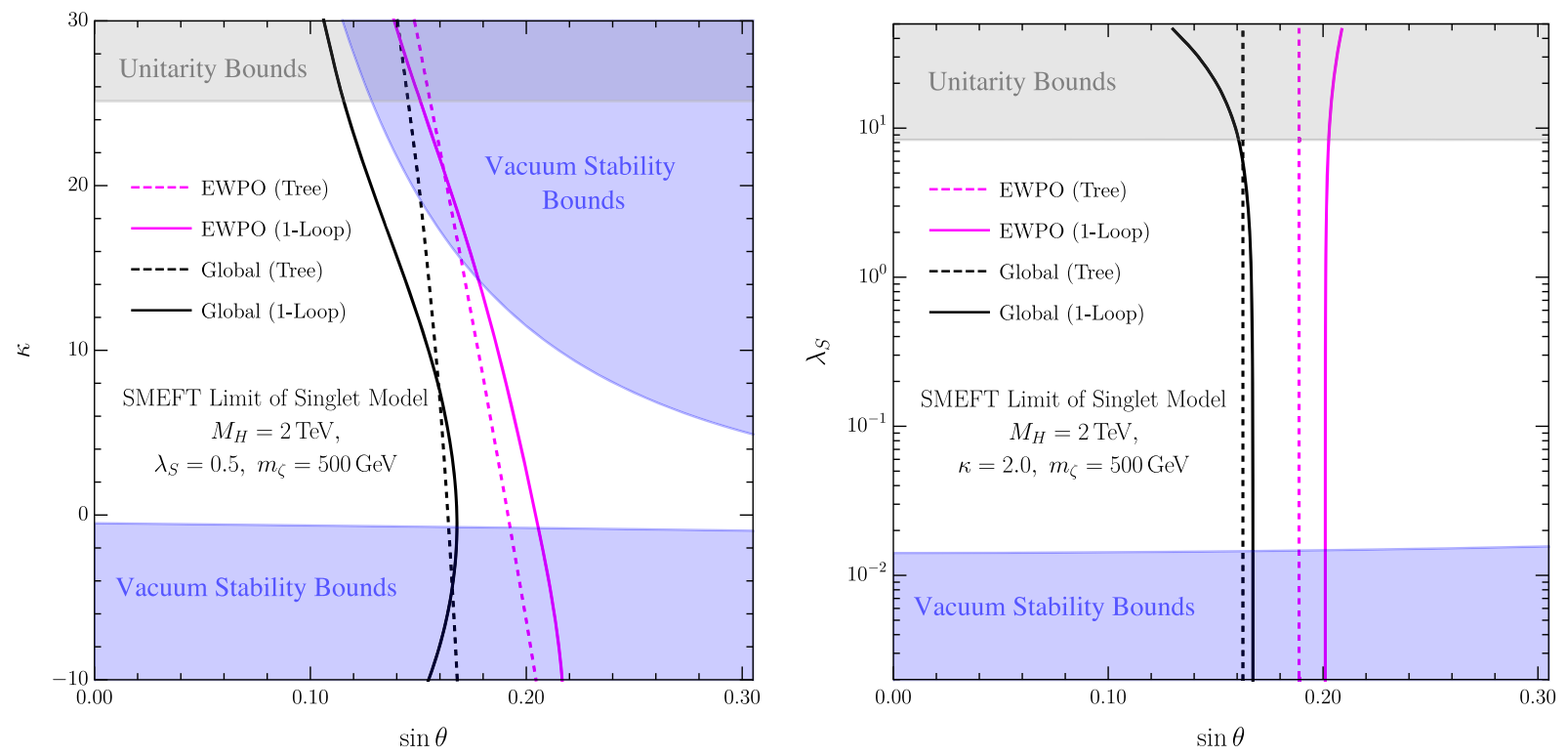

FIG. 7. $95 \%$ C.L. limits on $\sin \theta$ as a function of $\kappa$ (left) and $\lambda_{S}$ (right) for fixed $M_{H}=2 \mathrm{TeV}$ and $m_{\zeta}=500 \mathrm{GeV}$. The fits with treelevel matching are shown as dashed curves, with solid curves showing the one-loop result. The black curves show the result of a global fit to Higgs, diboson, and electroweak precision data, while the pink curves only the electroweak precision observables. The regions to the right of the curves are excluded by the fits. The grey and blue shaded regions are forbidden by unitarity and electroweak vacuum stability requirements, respectively.

calculated using Eq. (4). We match the SMEFT coefficients at $M$ and use the one-loop renormalization group equations to evolve the SMEFT coefficients to $M_{Z}$ where we fit to data.

The included data are identical to that of Ref. [28] and include Higgs coupling strengths from ATLAS [45], CMS Higgs coupling strengths [46], $W^{+} W^{-}, W^{ \pm} Z, W h$, and $Z h$ differential measurements including QCD effects as in

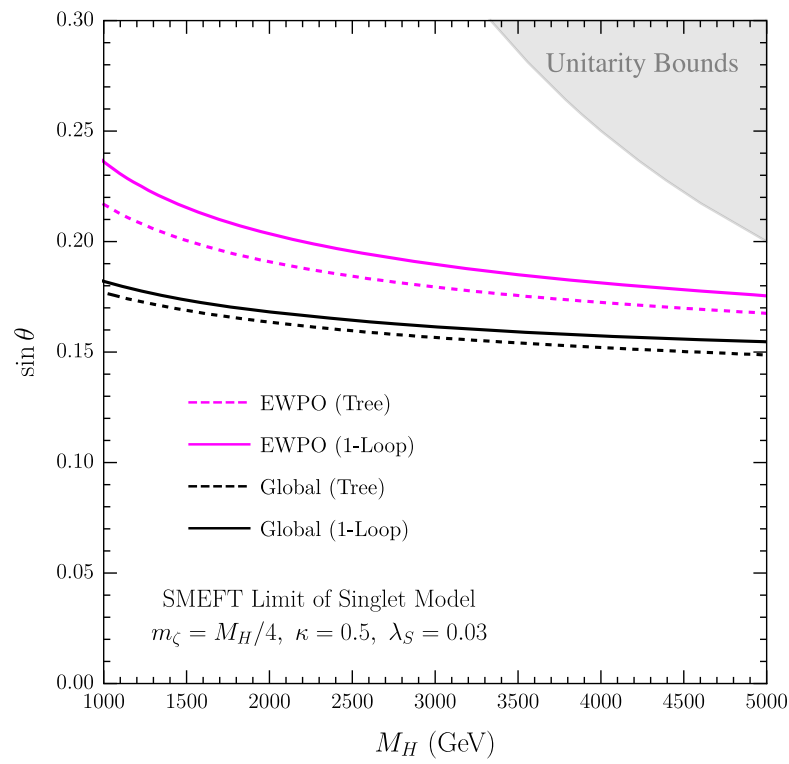

[78,79], and precision electroweak measurements including QCD and electroweak NLO effects from Table III of Ref. [54]. We determine the $95 \%$ confidence level limits using a $\chi^{2}$ fit, including the new physics effects at linear order in the SMEFT coefficients.

Figures 7 and 8 contain our major results. In terms of the parameters of the singlet model given in Eq. (3), we fix $M_{H}=2 \mathrm{TeV}$ and determine the maximum allowed value

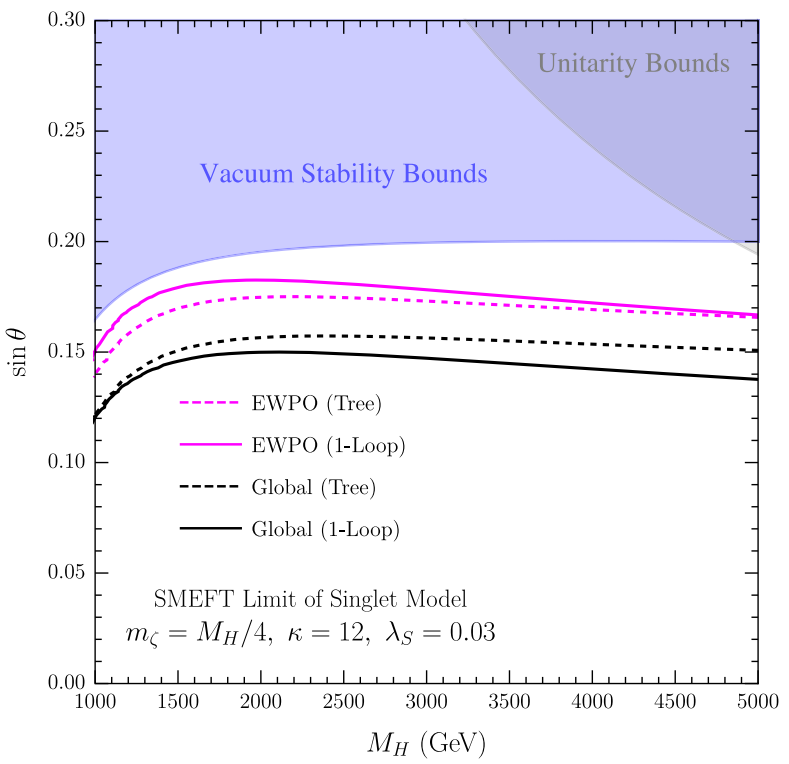

FIG. 8. As in Fig. 7, now showing limits on $\sin \theta$ as a function of the heavy Higgs mass, $M_{H}$, with fixed values of $\kappa, \lambda_{S}$, and $m_{\zeta} / M_{H}$. Regions above the curves are excluded. 
of $\sin \theta$ in terms of the other unknown parameters of the model, $\lambda_{S}, \kappa$, and $m_{\zeta}$. The curves are relatively insensitive to $m_{\zeta}$ and $\lambda_{S}$ (right-hand side), and the major sensitivity is to $\kappa$ (left-hand side) of Fig. 7. We show the regions excluded by unitarity bounds and by vacuum stability bounds. The black curves include the Higgs, diboson, and EWPO data. For $\kappa \lesssim 8$, the inclusion of the one-loop matching makes very little difference, but as $\kappa$ becomes large and approaches the unitarity bound, the difference between tree-level and one-loop matching can be of $\mathcal{O}(10 \%)$. We separately show the limits from only EWPO limits in magenta and note that the one-loop matching slightly improves the bound on $\sin \theta$.

Another interesting way to look at the results is to look at the maximum allowed value of $\sin \theta$ as a function of the heavy Higgs mass, $M_{H}$, for fixed values of $\kappa, m_{\zeta}$, and $\lambda_{S}$ as shown in Fig. 8. We see that including the one-loop matching changes the bound on $\sin \theta$ only marginally. The effect is larger as $\kappa$ is increased.

Single parameter fits to models with an additional scalar have been presented in Ref. [33] and updated in Ref. [34] using the dictionary of Ref. [72]. Assuming $C_{H \square}=-1 / M^{2}$ and $C_{H}=0$, they find a limit $M_{H}>900 \mathrm{GeV}$ at $2 \sigma$. Our tree-level matching result of Ref. [28] is roughly compatible with this bound, although we find that the inclusion of the renormalization group running of the coefficients (in particular $C_{H D}$ which is generated by renormalization group running) is numerically significant, so the bounds cannot be directly compared. ${ }^{11}$

\footnotetext{
${ }^{11}$ Fits to the singlet model with one-loop matching, but no renormalization group running, are given in Ref. [35], but the results are not in a form that we can compare with.
}

\section{CONCLUSIONS}

We have reexamined the sensitivity of a global fit to electroweak precision observables and to Higgs and diboson data on the parameters of a scalar singlet model in both the full UV complete model and in the low energy approximation where the heavy scalar is integrated out and the parameters are matched to the dimension- 6 SMEFT. In the full singlet model, we find equivalent limits on the allowed mixing angle from the complete EWPO fit and from the fit to the oblique parameters when $M_{H}$ is heavy. For the case with the second Higgs boson much lighter than $M_{Z}$, the oblique parameter limits are not a good approximation to the full fit. When the new scalar is very heavy, we integrate it out and match to the dimension6 SMEFT and then perform the global fit both using treelevel and one-loop matching at the high scale and derive limits on the parameters of the singlet theory from the SMEFT fit. We find that the effect on the fit of including the one-loop matching is never larger than $\mathcal{O}(10 \%)$ and that the results are quite insensitive to variations in the singlet Lagrangian parameters other than the portal term, $\kappa$.

Digital data can be found at [57].

\section{ACKNOWLEDGMENTS}

S. D. is supported by the U.S. Department of Energy under Grant Contract No. DE-SC0012704. The work of P. P. G. has received financial support from Xunta de Galicia (Centro singular de investigación de Galicia accreditation 2019-2022), by European Union ERDF, and by "María de Maeztu" Units of Excellence program No. MDM-2016-0692 and the Spanish Research State Agency. The work of S.H. was supported by DOE Grant No. DE-SC0013607 and by the Alfred P. Sloan Foundation Grant No. G-2019-12504.
[1] M. Bowen, Y. Cui, and J. D. Wells, Narrow trans-TeV Higgs bosons and $H \rightarrow h$ decays: Two LHC search paths for a hidden sector Higgs boson, J. High Energy Phys. 03 (2007) 036.

[2] D. O'Connell, M. J. Ramsey-Musolf, and M. B. Wise, Minimal extension of the standard model scalar sector, Phys. Rev. D 75, 037701 (2007).

[3] S. Dawson and I. M. Lewis, NLO corrections to double Higgs boson production in the Higgs singlet model, Phys. Rev. D 92, 094023 (2015).

[4] M. Mühlleitner, M. O. Sampaio, R. Santos, and J. Wittbrodt, ScannerS: Parameter scans in extended scalar sectors, arXiv:2007.02985.
[5] T. Robens and T. Stefaniak, Status of the Higgs singlet extension of the standard model after LHC Run 1, Eur. Phys. J. C 75, 104 (2015).

[6] R. Costa, M. Mühlleitner, M. O. P. Sampaio, and R. Santos, Singlet extensions of the standard model at LHC Run 2: Benchmarks and comparison with the NMSSM, J. High Energy Phys. 06 (2016) 034.

[7] C.-Y. Chen, S. Dawson, and I. M. Lewis, Exploring resonant di-Higgs boson production in the Higgs singlet model, Phys. Rev. D 91, 035015 (2015).

[8] S. J. Huber, T. Konstandin, T. Prokopec, and M. G. Schmidt, Electroweak phase transition and baryogenesis in the nMSSM, Nucl. Phys. B757, 172 (2006). 
[9] S. Profumo, M. J. Ramsey-Musolf, and G. Shaughnessy, Singlet Higgs phenomenology and the electroweak phase transition, J. High Energy Phys. 08 (2007) 010.

[10] J. R. Espinosa, T. Konstandin, and F. Riva, Strong electroweak phase transitions in the standard model with a singlet, Nucl. Phys. B854, 592 (2012).

[11] V. Barger, D. J. H. Chung, A. J. Long, and L.-T. Wang, Strongly first order phase transitions near an enhanced discrete symmetry point, Phys. Lett. B 710, 1 (2012).

[12] S. Profumo, M. J. Ramsey-Musolf, C. L. Wainwright, and P. Winslow, Singlet-catalyzed electroweak phase transitions and precision Higgs boson studies, Phys. Rev. D 91, 035018 (2015).

[13] D. Curtin, P. Meade, and C.-T. Yu, Testing electroweak baryogenesis with future colliders, J. High Energy Phys. 11 (2014) 127.

[14] A. V. Kotwal, M. J. Ramsey-Musolf, J. M. No, and P. Winslow, Singlet-catalyzed electroweak phase transitions in the $100 \mathrm{TeV}$ frontier, Phys. Rev. D 94, 035022 (2016).

[15] T. Huang, J. M. No, L. Pernie, M. Ramsey-Musolf, A. Safonov, M. Spannowsky, and P. Winslow, Resonant diHiggs boson production in the $b \bar{b} W W$ channel: Probing the electroweak phase transition at the LHC, Phys. Rev. D 96, 035007 (2017).

[16] C.-Y. Chen, J. Kozaczuk, and I. M. Lewis, Non-resonant collider signatures of a singlet-driven electroweak phase transition, J. High Energy Phys. 08 (2017) 096.

[17] G. Kurup and M. Perelstein, Dynamics of electroweak phase transition in singlet-scalar extension of the standard model, Phys. Rev. D 96, 015036 (2017).

[18] H.-L. Li, M. Ramsey-Musolf, and S. Willocq, Probing a scalar singlet-catalyzed electroweak phase transition with resonant di-Higgs boson production in the $4 b$ channel, Phys. Rev. D 100, 075035 (2019).

[19] N. Craig, C. Englert, and M. McCullough, New Probe of Naturalness, Phys. Rev. Lett. 111, 121803 (2013).

[20] D. Curtin and P. Saraswat, Towards a no-lose theorem for naturalness, Phys. Rev. D 93, 055044 (2016).

[21] V. Silveira and A. Zee, Scalar phantoms, Phys. Lett. 161B, 136 (1985).

[22] J. McDonald, Gauge singlet scalars as cold dark matter, Phys. Rev. D 50, 3637 (1994).

[23] C. P. Burgess, M. Pospelov, and T. ter Veldhuis, The minimal model of nonbaryonic dark matter: A singlet scalar, Nucl. Phys. B619, 709 (2001).

[24] A. Menon, D. E. Morrissey, and C. E. M. Wagner, Electroweak baryogenesis and dark matter in the nMSSM, Phys. Rev. D 70, 035005 (2004).

[25] X.-G. He, T. Li, X.-Q. Li, J. Tandean, and H.-C. Tsai, Constraints on scalar dark matter from direct experimental searches, Phys. Rev. D 79, 023521 (2009).

[26] M. Gonderinger, Y. Li, H. Patel, and M. J. Ramsey-Musolf, Vacuum stability, perturbativity, and scalar singlet dark matter, J. High Energy Phys. 01 (2010) 053.

[27] Y. Mambrini, Higgs searches and singlet scalar dark matter: Combined constraints from XENON 100 and the LHC, Phys. Rev. D 84, 115017 (2011).

[28] S. Dawson, S. Homiller, and S. D. Lane, Putting standard model EFT fits to work, Phys. Rev. D 102, 055012 (2020).
[29] J. Brehmer, A. Freitas, D. Lopez-Val, and T. Plehn, Pushing Higgs effective theory to its limits, Phys. Rev. D 93, 075014 (2016).

[30] B. Henning, X. Lu, and H. Murayama, What do precision Higgs measurements buy us?, arXiv:1404.1058.

[31] B. Henning, X. Lu, and H. Murayama, How to use the standard model effective field theory, J. High Energy Phys. 01 (2016) 023.

[32] M. Gorbahn, J. M. No, and V. Sanz, Benchmarks for Higgs effective theory: Extended Higgs sectors, J. High Energy Phys. 10 (2015) 036.

[33] J. Ellis, C. W. Murphy, V. Sanz, and T. You, Updated global SMEFT fit to Higgs, diboson and electroweak data, J. High Energy Phys. 06 (2018) 146.

[34] J. Ellis, M. Madigan, K. Mimasu, V. Sanz, and T. You, Top, Higgs, diboson and electroweak fit to the standard model effective field theory, arXiv:2012.02779.

[35] Anisha, S. Das Bakshi, J. Chakrabortty, and S. K. Patra, A step toward model comparison: Connecting electroweakscale observables to BSM through EFT and Bayesian statistics, arXiv:2010.04088.

[36] D. Egana-Ugrinovic and S. Thomas, Effective theory of Higgs sector vacuum states, arXiv:1512.00144.

[37] S. Das Bakshi, J. Chakrabortty, and M. Spannowsky, Classifying Standard Model extensions effectively with precision observables, arXiv:2012.03839.

[38] G. D. Kribs, A. Maier, H. Rzehak, M. Spannowsky, and P. Waite, Electroweak oblique parameters as a probe of the trilinear Higgs boson self-interaction, Phys. Rev. D 95, 093004 (2017).

[39] A. Falkowski, C. Gross, and O. Lebedev, A second Higgs from the Higgs portal, J. High Energy Phys. 05 (2015) 057.

[40] M. Jiang, N. Craig, Y.-Y. Li, and D. Sutherland, Complete one-loop matching for a singlet scalar in the standard model EFT, J. High Energy Phys. 02 (2019) 031; Erratum, J. High Energy Phys. 01 (2021) 135.

[41] U. Haisch, M. Ruhdorfer, E. Salvioni, E. Venturini, and A. Weiler, Singlet night in Feynman-ville: One-loop matching of a real scalar, J. High Energy Phys. 04 (2020) 164; Erratum, J. High Energy Phys. 07 (2020) 066.

[42] T. Cohen, X. Lu, and Z. Zhang, Functional prescription for EFT matching, J. High Energy Phys. 02 (2021) 228.

[43] G. Buchalla, O. Cata, A. Celis, and C. Krause, Standard model extended by a heavy singlet: Linear vs nonlinear EFT, Nucl. Phys. B917, 209 (2017).

[44] S. Dawson, A. Ismail, and I. Low, What's in the loop? The anatomy of double Higgs production, Phys. Rev. D 91, 115008 (2015).

[45] G. Aad et al. (ATLAS Collaboration), Combined measurements of Higgs boson production and decay using up to $80 \mathrm{fb}^{-1}$ of proton-proton collision data at $\sqrt{s}=13 \mathrm{TeV}$ collected with the ATLAS experiment, Phys. Rev. D 101, 012002 (2020).

[46] CMS Collaboration, Combined Higgs boson production and decay measurements with up to $137 \mathrm{fb}^{-1}$ of proton-proton collision data at $\sqrt{s}=13 \mathrm{TeV}$, https://cds.cern.ch/record/ 2706103.

[47] A. Ilnicka, T. Robens, and T. Stefaniak, Constraining extended scalar sectors at the LHC and beyond, Mod. Phys. Lett. A 33, 1830007 (2018). 
[48] N. Kauer and C. O'Brien, Heavy Higgs signal-background interference in $g g \rightarrow V V$ in the standard model plus real singlet, Eur. Phys. J. C 75, 374 (2015).

[49] N. Kauer, C. O'Brien, and E. Vryonidou, Interference effects for $H \rightarrow W W \rightarrow \ell \nu q \bar{q}^{\prime}$ and $H \rightarrow Z Z \rightarrow \ell \bar{\ell} q \bar{q}$ searches in gluon fusion at the LHC, J. High Energy Phys. 10 (2015) 074.

[50] N. Kauer, A. Lind, P. Maierhöfer, and W. Song, Higgs interference effects at the one-loop level in the 1-Higgssinglet extension of the standard model, J. High Energy Phys. 07 (2019) 108.

[51] G. Aad et al. (ATLAS Collaboration), Combination of searches for Higgs boson pairs in $p p$ collisions at $\sqrt{s}=$ $13 \mathrm{TeV}$ with the ATLAS detector, Phys. Lett. B 800, 135103 (2020).

[52] W. F. L. Hollik, Radiative corrections in the standard model and their role for precision tests of the electroweak theory, Fortschr. Phys. 38, 165 (1990).

[53] A. Freitas, Higher-order electroweak corrections to the partial widths and branching ratios of the $\mathrm{Z}$ boson, J. High Energy Phys. 04 (2014) 070.

[54] S. Dawson and P. P. Giardino, Electroweak and QCD corrections to $Z$ and $W$ pole observables in the standard model EFT, Phys. Rev. D 101, 013001 (2020).

[55] S. Carrazza, R. K. Ellis, and G. Zanderighi, QCDLoop: A comprehensive framework for one-loop scalar integrals, Comput. Phys. Commun. 209, 134 (2016).

[56] D. Lopez-Val and T. Robens, $\Delta r$ and the W-boson mass in the singlet extension of the standard model, Phys. Rev. D 90, 114018 (2014).

[57] S. Dawson, P. P. Giardino, and S. Homiller, https://quark .phy.bnl.gov/Digital_Data_Archive/dawson/singlet_21.

[58] S. Dawson and W. Yan, Hiding the Higgs boson with multiple scalars, Phys. Rev. D 79, 095002 (2009).

[59] C. Englert, J. Jaeckel, M. Spannowsky, and P. Stylianou, Power meets precision to explore the symmetric Higgs portal, Phys. Lett. B 806, 135526 (2020).

[60] M.E. Peskin and T. Takeuchi, Estimation of oblique electroweak corrections, Phys. Rev. D 46, 381 (1992).

[61] P. Zyla et al. (Particle Data Group), Review of particle physics, Prog. Theor. Exp. Phys. 2020, 083 C01 (2020).

[62] G. Chalons, D. Lopez-Val, T. Robens, and T. Stefaniak, The Higgs singlet extension at LHC Run 2, Proc. Sci., DIS2016 (2016) 113.

[63] J. de Blas, M. Ciuchini, E. Franco, S. Mishima, M. Pierini, L. Reina, and L. Silvestrini, The global electroweak and Higgs fits in the LHC era, Proc. Sci., EPS-HEP2017 (2017) 467.

[64] M. Aaboud et al. (ATLAS Collaboration), Search for Higgs boson decays into a pair of light bosons in the $b b \mu \mu$ final state in $p p$ collision at $\sqrt{s}=13 \mathrm{TeV}$ with the ATLAS detector, Phys. Lett. B 790, 1 (2019).
[65] S. Choudhury (CMS Collaboration), Searches for Light Higgs Bosons at the CMS Experiment, Technical Report No. CMS-CR-2018-260, CERN, Geneva, 2018, https://cds .cern.ch/record/2644442.

[66] T. Robens and T. Stefaniak, LHC benchmark scenarios for the real Higgs singlet extension of the standard model, Eur. Phys. J. C 76, 268 (2016).

[67] B. W. Lee, C. Quigg, and H. Thacker, The Strength of Weak Interactions at Very High-Energies and the Higgs Boson Mass, Phys. Rev. Lett. 38, 883 (1977).

[68] B. W. Lee, C. Quigg, and H. Thacker, Weak interactions at very high energies: The role of the Higgs boson mass, Phys. Rev. D 16, 1519 (1977).

[69] S. Dawson and C. W. Murphy, standard model EFT and extended scalar sectors, Phys. Rev. D 96, 015041 (2017).

[70] W. Buchmuller and D. Wyler, Effective Lagrangian analysis of new interactions and flavor conservation, Nucl. Phys. B268, 621 (1986).

[71] A. Dedes, W. Materkowska, M. Paraskevas, J. Rosiek, and K. Suxho, Feynman rules for the standard model effective field theory in $R_{\xi}$-gauges, J. High Energy Phys. 06 (2017) 143.

[72] J. de Blas, J. C. Criado, M. Perez-Victoria, and J. Santiago, Effective description of general extensions of the standard model: The complete tree-level dictionary, J. High Energy Phys. 03 (2018) 109.

[73] E. E. Jenkins, A. V. Manohar, and M. Trott, Renormalization group evolution of the standard model dimension six operators. I: Formalism and lambda dependence, J. High Energy Phys. 10 (2013) 087.

[74] E. E. Jenkins, A. V. Manohar, and M. Trott, Renormalization group evolution of the standard model dimension six operators. II: Yukawa dependence, J. High Energy Phys. 01 (2014) 035.

[75] R. Alonso, E. E. Jenkins, A. V. Manohar, and M. Trott, Renormalization group evolution of the standard model dimension six operators. III: Gauge coupling dependence and phenomenology, J. High Energy Phys. 04 (2014) 159.

[76] G. Degrassi, M. Fedele, and P. P. Giardino, Constraints on the trilinear Higgs self coupling from precision observables, J. High Energy Phys. 04 (2017) 155.

[77] G. Degrassi, P. P. Giardino, F. Maltoni, and D. Pagani, Probing the Higgs self coupling via single Higgs production at the LHC, J. High Energy Phys. 12 (2016) 080.

[78] J. Baglio, S. Dawson, and S. Homiller, QCD corrections in standard model EFT fits to $W Z$ and $W W$ production, Phys. Rev. D 100, 113010 (2019).

[79] J. Baglio, S. Dawson, S. Homiller, S. D. Lane, and I. M. Lewis, Validity of standard model EFT studies of VH and VV production at NLO, Phys. Rev. D 101, 115004 (2020). 